\title{
AFEL - Analytics for Everyday Learning
}

\author{
Mathieu d'Aquin, Dominik Kowald, Angela Fessl, Elisabeth Lex, Stefan Thalmann \\ mathieu.daquin@insight-centre.org, Insight Centre for Data Analytics, NUI Galway (Ireland) \\ \{afessl,dkowald,elex,sthalmann\}@know-center.at, Know-Center GmbH Graz (Austria)
}

\begin{abstract}
The goal of AFEL is to develop, pilot and evaluate methods and applications, which advance informal/collective learning as it surfaces implicitly in online social environments. The project is following a multi-disciplinary, industry-driven approach to the analysis and understanding of learner data in order to personalize, accelerate and improve informal learning processes. Learning Analytics and Educational Data Mining traditionally relate to the analysis and exploration of data coming from learning environments, especially to understand learners' behaviours. However, studies have for a long time demonstrated that learning activities happen outside of formal educational platforms, also. This includes informal and collective learning usually associated, as a side effect, with other (social) environments and activities. Relying on real data from a commercially available platform, the aim of AFEL is to provide and validate the technological grounding and tools for exploiting learning analytics on such learning activities. This will be achieved in relation to cognitive models of learning and collaboration, which are necessary to the understanding of loosely defined learning processes in online social environments. Applying the skills available in the consortium to a concrete set of live, industrial online social environments, AFEL will tackle the main challenges of informal learning analytics through 1) developing the tools and techniques necessary to capture information about learning activities from (not necessarily educational) online social environments; 2) creating methods for the analysis of such informal learning data, based on combining feature engineering and visual analytics with cognitive models of learning and collaboration; and 3) demonstrating the potential of the approach in improving the understanding of informal learning, and the way it is better supported; 4) evaluate all the former items in real world large scale applications and platforms.
\end{abstract}

\section{KEYWORDS}

AFEL; Learning Analytics; Everyday Learning; H2020

\section{ACM Reference Format:}

Mathieu d'Aquin, Dominik Kowald, Angela Fessl, Elisabeth Lex, Stefan Thalmann. 2018. AFEL - Analytics for Everyday Learning. In WWW' 18 Companion: The 2018 Web Conference Companion, April 23-27, 2018, Lyon, France. ACM, New York, NY, USA, 2 pages. https://doi.org/10.1145/3184558.3186206

This paper is published under the Creative Commons Attribution 4.0 International (CC BY 4.0) license. Authors reserve their rights to disseminate the work on their personal and corporate Web sites with the appropriate attribution.

WWW'18 Companion, April 23-27, 2018, Lyon, France

() 2018 IW3C2 (International World Wide Web Conference Committee), published under Creative Commons CC BY 4.0 License.

ACM ISBN 978-1-4503-5640-4/18/04.

https://doi.org/10.1145/3184558.3186206

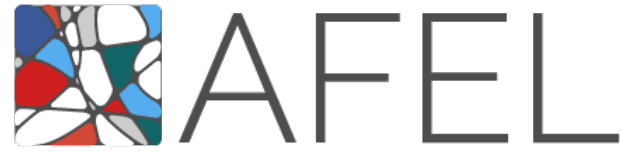

Figure 1: Logo of the H2020 Analytics for the Everyday Learning (AFEL) project.

\section{BASIC PROJECT INFORMATION}

\section{Consortium.}

- Data Science Institute, Insight Centre for Data Analytics, NUI Galway (Ireland): Mathieu d'Aquin, Coordinator

- Gnoss, Riam Intelearning Lab S.L (Spain): Ricardo Maturana and Esteban Sota

- Know-Center GmbH Graz (Austria): Elisabeth Lex, Dominik Kowald, Eduardo Veas, Ilire Hasani-Mavriqi, Angela Fessl and Stefan Thalmann

- Knowledge Media Research Center, Stiftung Medien in der Bildung (Germany): Joachim Kimmerle and Peter Holtz

- Leibniz University Hannover (Germany): Stefan Dietze and Besnik Fetahu

- The Open University (UK): Ilaria Tiddi and Alessandro Adamou

Name of the presenter. Mathieu d'Aquin, Data Science Institute, Insight Centre for Data Analytics, NUI Galway (Ireland)

Project duration. From 2015-12-01 to 2018-11-30

Project volume. 2,581,940 euro

Funding agency. European Union's Horizon 2020 research and innovation programme under grant agreement No 687916

Official Web site. http://afel-project.eu

\section{WHY THIS RESEARCH IS IMPORTANT}

In several other areas than learning where self-directed activities are prominent (e.g. fitness), there has been a trend in recent years following the technological development of tools for self-tracking. Those tools quantify a specific user's activities with respect to a certain goal (e.g. being physically fit) to enable self-awareness and reflection, with the purpose of turning them into behavioral changes. While the actual benefits of self-tracking in those areas are still debatable, our understanding of how such approaches could benefit learning behaviors as they become self-directed remains limited.

AFEL (see Figure 1) aims is to address both the theoretical and technological challenges arising from applying Learning Analytics in the context of online, social learning [1]. The pillars of the project are the technologies to capture large scale, heterogeneous data about learners' online activities across multiple platforms (including 
Social Media) and the operationalization of theoretical cognitive models of learning to measure and assess those online learning activities.

\section{CONTENT OF THE PRESENTATION AND DEMONSTRATION}

The presentation will describe the results obtained in the first two years of the project. This includes a large data platform designed to collected, integrate and redistribute data about user activities and the online resources they use to learn. This will be demonstrated both in the context of the Didactalia online learning platform provided by AFEL partner GNOSS (including hundreds of thousands of users, using tens of thousands if resources), as well as in the more generally of learning using online resources from various platform (online videos, MOOCs, Social Media, etc).

Based on the data collected, we will show a variety of techniques employed to integrate and semantically enrich those data so to compute indicators of the progress and effectiveness of the online learning behaviour of the user. Those indicators are tracked through a mobile application and dashboards providing a range of visual analytics tools targeted at both novice and advanced users. Those applications will be demonstrated during the presentation using real data.

\section{RESULTS}

In its first two years, the project has had a number of significant results, including demos of the various aspects of the project, papers on the different aspects of the research (including data integration, enrichment, visualisation and the cognitive model of learning). We summarize below four of the most significant results:

AFEL data platform. The data platform on which AFEL relies is built to enable ingesting data from a large number of heterogeneous sources, so to enable aggregating those data, enriching them, and providing them back through APIs available to learner-facing tools. A key challenge is to ensure that the data is connected around a user identified differently on different platforms. We rely on a core data schema to remodel the data in RDF, as well as on the ElasticSearch platform to handle large volume of activity data. More information: http://data.afel-project.eu/.

AFEL visual analytics framework. Through the visual analytics framework developed in the project, it is possible to create flexible dashboards enabling a large number of automatically recommended customisable visualisations. More information: http: //analytics.didactalia.net

AFEL open datasets. As Learning Analytics researchers, we use data which can also be of use to other researchers. The AFEL Dataset for Learning Analytics is a collection of data sources that are useful for performing analytics in online/social learning contexts. It is distilled from the content of the AFEL Data Catalogue, excluding datasets containing personal data and others that are not freely redistributable. It aggregates over 38B distinct RDF triples, obtained both by refactoring existing linked datasets and by reengineering third-party datasets. More information: http://data.afelproject.eu/catalogue/dataset-for-learning-analytics-v2/
Learning analytics glossary. Learning Analytics requires to manipulate complex notions related to the cognitive processes of learning, and make them computationally operational through analytics processes. The Learning Analytics Glossary is an AFEL-led collaborative initiative to establish shared definitions for those notions, as well as approaches to approximate them in Learning Analytics, focusing on social, self-directed learning. More information: http://laglossary.github.io

Learning resource recommender system. The resource recommendation services aim to provide suggestions of learning resources to users in Didactalia. These recommendations are calculated based on the identified and extracted features indicative of learning activities such as past user interactions, resource popularity and resource content. Three use cases have been identified that are of interest for the project: (i) recommendation of popular resources in the system (i.e., non-personalized), (ii) recommendation of resources with whom like-minded users have interacted (i.e, personalized), (iii) recommendation of (alternative) similar resources for a specific resource (i.e., more-like-this). For the implementation, we will build upon our previous research in this area (e.g., [2-4]) as well as utilize our scalable recommendation framework ScaR (e.g., [5]). More information: http://afel-project.eu/toolkit/afel-recommender/

\section{ACKNOWLEDGMENTS}

The authors would like to thank the rest of the AFEL consortium. This work was supported by the Know-Center Graz, the Science Foundation Ireland (SFI) Insight Centre for Data Analytics and the European-funded project AFEL (GA687916). The Know-Center Graz is funded within the Austrian COMET Program - Competence Centers for Excellent Technologies - under the auspices of the Austrian Ministry of Transport, Innovation and Technology, the Austrian Ministry of Economics and Labor and by the State of Styria. COMET is managed by the Austrian Research Promotion Agency (FFG).

\section{REFERENCES}

[1] Mathieu d'Aquin, Alessandro Adamou, Stefan Dietze, Besnik Fetahu, Ujwal Gadiraju, Ilire Hasani-Mavriqi, Peter Holtz, Joachim Kimmerle, Dominik Kowald, and Elisabeth Lex. 2017. AFEL: Towards Measuring Online Activities Contributions to Self-Directed Learning. In Proceedings of Proceedings of the 7th Workshop on Awareness and Reflection in Technology Enhanced Learning (ARTEL) in conjunction with the 12th European Conference on Technology Enhanced Learning: Adaptive and Adaptable Learning (EC-TEL 2017).

[2] Dominik Kowald, Simone Kopeinik, and Elisabeth Lex. 2017. The TagRec Framework As a Toolkit for the Development of Tag-Based Recommender Systems. In Adjunct Publication of the 25th Conference on User Modeling, Adaptation and Personalization (UMAP '17). ACM, New York, NY, USA, 23-28. https: //doi.org/10.1145/3099023.3099069

[3] Dominik Kowald and Elisabeth Lex. 2015. Evaluating tag recommender algorithms in real-world folksonomies: A comparative study. In Proceedings of the 9th ACM Conference on Recommender Systems. ACM, 265-268.

[4] Dominik Kowald, Subhash Chandra Pujari, and Elisabeth Lex. 2017. Temporal Effects on Hashtag Reuse in Twitter: A Cognitive-Inspired Hashtag Recommendation Approach. In Proceedings of the 26th International Conference on World Wide Web (WWW '17). International World Wide Web Conferences Steering Committee, Republic and Canton of Geneva, Switzerland, 1401-1410. https://doi.org/10.1145/3038912.3052605

[5] Emanuel Lacic, Matthias Traub, Dominik Kowald, and Elisabeth Lex. 2017. ScaR: Towards a Real-Time Recommender Framework Following the Microservices Architecture. In Proceedings of the Workshop on Large Scale Recommender Systems (LSRS'2015) co-located with the 9th ACM Conference on Recommender Systems (RecSys'2015). 